\title{
Tolerance Stack Analysis in Francis Turbine Design
}

\author{
Indra Djodikusumo, Koko Suherman \& Paskalis Bowo A. Oken
}

\author{
Faculty of Mechanical Engineering and Aerospace, \\ Institute of Technology Bandung \\ Email: djodikusumo.indra@gmail.com
}

\begin{abstract}
The tolerance stacking problem arises in the context of assemblies from interchangeable parts because of the inability to produce or to join parts exactly according to nominal dimensions. Either the relevant part's dimension varies around some nominal values from part to part or the act of assembly that leads to variation. For example, as runner of Francis turbine is joined with turbine shaft via mechanical lock, there is not only variation in the diameter of runner and the concentricity between the runner hole and turbine shaft, but also the variation in concentricity between the outer parts of runner to runner hole. Thus, there is the possibility that the assembly of such interacting parts won't function or won't come together as planned. Research in this area has been conducted and 2 mini hydro Francis turbines $(800 \mathrm{~kW}$ and $910 \mathrm{~kW})$ have been designed and manufactured for San Sarino and Sawi Dago 2 in Central Sulawesi. Experiences in analyzing the tolerance stacks have been documented. In this paper it will be demonstrated how the requirements of assembling performance are derived to be the designed tolerances of each interacting component, such a way that the assembling would be functioning and come together as planned.
\end{abstract}

Keywords: assembling requirements; geometric dimensioning and tolerancing; tolerance stacks; variation of feature geometry; worst case method.

\section{Introduction}

The utilization of renewable energy as an alternative to fosil energy has been promoted all around the world, including Indonesia. Since then, the demand of mini hydro turbines in Indonesia increases very fast. Around 40 mini hydro turbines are required in Indonesia each year until 2020 [1]. The power for the required turbines is around $500 \mathrm{~kW}$ until $2 \mathrm{MW}$. Most of them are Francis type and some others are from other types such as Kaplan for low head potential sites and Pelton for high head potential sites. This is a very good opportunity for Indonesia to develop their own mini hydro power plant using their capacity, not only in the engineering, procurement and construction (EPC), but also in the supply of the required equipments such as turbines, generators, control panels, transformers and others. PT. Ganesha Reverse Engineering and Toolmaking (GREAT) is one of the tenant in Industry and Business Incubator at the Institute of Technology Bandung, that design mini hydro turbines, especially Francis, Kaplan and Pelton Turbines. With some partners in the manufacturing industry,

Received December $10^{\text {th }}, 2009$, Revised March $17^{\text {th }}, 2010$, Accepted for publication March $20^{\text {th }}, 2010$. 
mostly small and medium size companies, PT. GREAT starts manufacturing mini hydro turbines. Eventhough it is not easy to penetrate the market, some companies such as the National Electricity Provider (PT. PLN) starts ordering mini hydro turbines from PT. GREAT. It is the objectives of PT. GREAT to produce more mini hydro turbines that fulfill some criterias as follows:

- Good performance

- Good relliability

- Competitive delivery time

- Competitive price

- Ease of operation

- $\quad$ Ease of maintenance

- Good after sale service by providing its components using part number, so the concept of interchangeability should be implemented in the design and manufacturing

Many efforts have been conducted by PT. GREAT during the development of mini hydro turbines, and one of them is in the field of Geometric Dimensioning and Tolerancing (GDT). In order to achieve good efficiency, the losses especially due to water leakage should be avoided. Water should flow through runner blades, not through the gaps between runner and its covers and also between guide vanes and its covers. As the result, a very small gaps between runner and covers and between guide vanes and covers are required [2-6]. This small gaps require small tolerances for the geometric of its related turbine component features. Moreover, the objective to achieve the interchangeability of its spare parts using part number by after sales service also require the GDT theory. The GDT method that has been utilized for analyzing the tolerance stacks will be demonstrated and some of its results will be shown in this paper.

\section{Stacked Tolerance Analysis Methodology}

Stacked tolerance analysis is the process of breaking down components in assembly in order to take known tolerances each component and analyzing the combination of these tolerances at an assembly level. This analysis is done only at critical features in assembly.

The first step in the process is to identify the requirements for the system or we can say to identify features that have big contribution for fit and function of the product. These features are said critical for assembly. 
This stacked tolerance analysis will be divided into dimensional tolerance analysis and geometrical tolerance analysis.

\subsection{Dimensional Tolerance Analysis}

According to Paul Drake [7], the process of analysis for tolerance stacks could follow the following traditional approach (Figure 1). Each activity will be presented into more detail in the following sub chapter.

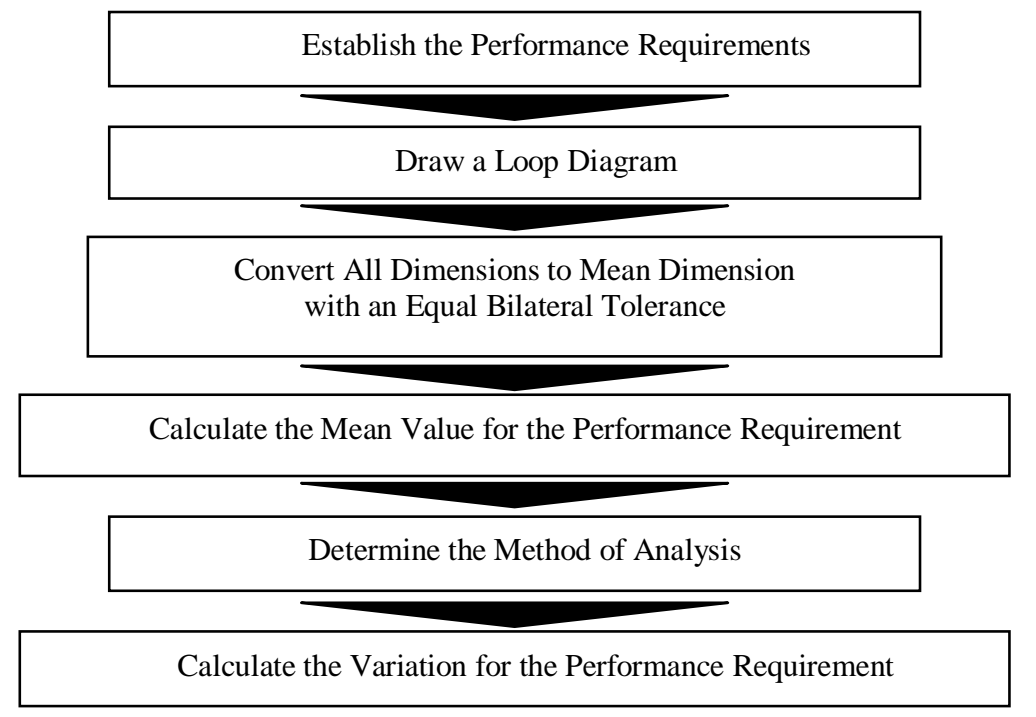

Figure 1 Tolerance Stack Analysis Process [7].

\subsubsection{Establish the Performance Requirements}

First, identify all the requirements for assembly system that will lead to the success of product's performance or ease of assembly. Then, flow down these requirements to each component. Finally, convert all performance requirements into gap requirements for assembly.

\subsubsection{Drawing of a Loop Diagram}

The loop diagram is a graphical representation of each analysis. Each requirement requires a separate loop diagram. There are two types of loop diagram, vertical and horizontal. Laws for drawing loop diagram are:

- For horizontal dimension loops, start at the surface on the left of the gap. Follow a complete dimension loop, to the surface on the right. For vertical dimension loops, start at the surface on the bottom of the gap. Follow a complete dimension loop, to the surface on the top. 
- Using vectors, create a closed loop diagram from the starting surface to the ending surface. Do not include gaps when selecting the path for the dimension loop. Each vector in the loop diagram represents a dimension.

- Use an arrow to show the direction of each vector in the dimension loop. Identify each vector as positive for dimension followed from left to right or from bottom to top, and as negative for dimension followed from right to left or from top to bottom.

- Assign a variable name to each dimension in the loop.

- Record sensitivities for each dimension. The magnitude of the sensitivity is the value that the gap changes, when the dimension changes 1 unit. For example, if the gap changes $1 \mathrm{~mm}$ when the dimension changes $1 \mathrm{~mm}$, then the magnitude of sensitivity is $1(1 \mathrm{~mm} / 1 \mathrm{~mm})$. On the other hand, if the gap changes $0.5 \mathrm{~mm}$ when the dimension changes $1 \mathrm{~mm}$, then the magnitude of sensitivity is $0.5(0.5 \mathrm{~mm} / 1 \mathrm{~mm})$. Usually, the magnitude of sensitivity is 0.5 for components involving diameter.

- Determine whether each dimension is fixed or variable. A fix dimension is one in which we have no control, such as a vendor part dimension. A variable dimension is one that we can change to influence the outcome of the tolerance stack, such as custom made components (made by order).

\subsubsection{Converting All Dimensions to Mean Dimension with an Equal Bilateral Tolerance}

Next, all tolerances in loop diagram should be change into equal bilateral tolerance, where upper and lower tolerance are equal. As a rule, designer should use equal bilateral tolerances, except if using this equal bilateral tolerances may force manufacturing to use nonstandard tools.

This change is based on fact that manufacturing process are normally distributed, where manufactured product's dimension will vary around its mean nominal dimensions. If the designer uses uniteral tolerances for the products, then most of products will be rejected. Steps for converting to an equal bilateral tolerance are:

- Calculate the upper and lower limit dimension.

- Subtract the lower limit from the upper limit to get the total tolerance band, and then divide the tolerance band by two to get an equal bilateral tolerance.

- Add the equal bilateral tolerance to the lower limit to get the mean dimension. Alternately, subtract the equal bilateral tolerance from the upper limit. 


\subsubsection{Calculate the Mean Value for the Performance Requirement}

The mean value of the requirements (gap) is calculated by [7-9]:

$$
d_{g}=\sum_{i=1}^{n} a_{i} d_{i}
$$

\subsubsection{Determine the Method of Analysis}

There are 3 types of tolerancing models to analyze the variation at the gap, which are worst case (WC) model, root sum of the square (RSS) statistical model and the combination of WC and RSS that is modified root sum of the square (MRSS) statistical model. WC model verifies all components will perform their intended function $100 \%$ of the time. This is a conservative approach and used for retail production or production by order. RSS model assume that most of the manufactured parts all centered on the mean dimension. This is used for mass production. MRSS model is created to bridge WC model which is too tight with RSS model which is too loose. The comparison of these 3 models can be seen at Table 1 .

Table 1 Comparison of Analysis Models [7].

\begin{tabular}{|c|c|c|c|}
\hline Consideration & WC Model & RSS Model & MRSS Model \\
\hline Risk of defect & Lowest & Highest & Middle \\
\hline Cost & Highest & Lowest & Middle \\
\hline $\begin{array}{l}\text { Assumptions } \\
\text { about } \\
\text { component } \\
\text { processes }\end{array}$ & None & $\begin{array}{l}\text { The process follows a } \\
\text { normal distribution. The } \\
\text { mean of the process is } \\
\text { equal to the nominal } \\
\text { dimension. Processes are } \\
\text { independent. }\end{array}$ & $\begin{array}{l}\text { The process follows a } \\
\text { normal distribution. The } \\
\text { mean of the process is not } \\
\text { necessarily equal to the } \\
\text { nominal dimension. }\end{array}$ \\
\hline $\begin{array}{l}\text { Assumptions } \\
\text { about drawing } \\
\text { tolerances }\end{array}$ & $\begin{array}{l}\text { Dimensions } \\
\text { outside the } \\
\text { tolerance range are } \\
\text { screened out. }\end{array}$ & $\begin{array}{l}\text { The tolerance is related to } \\
\text { manufacturing process } \\
\text { capability. Usually the } \\
\text { tolerance range is assume } \\
\text { to be the }+/-3 \sigma \text { limit of } \\
\text { the process. }\end{array}$ & $\begin{array}{l}\text { The tolerance is related to } \\
\text { manufacturing process } \\
\text { capability. Usually the } \\
\text { tolerance range is assume } \\
\text { to be the }+/-3 \sigma \text { limit of } \\
\text { the process. }\end{array}$ \\
\hline $\begin{array}{l}\text { Assumption } \\
\text { about expected } \\
\text { assembly } \\
\text { variation }\end{array}$ & $\begin{array}{l}100 \% \text { of the parts } \\
\text { are within the } \\
\text { maximum and } \\
\text { minimum } \\
\text { performance range. }\end{array}$ & $\begin{array}{l}\text { Assembly distribution is } \\
\text { normal. } 99.73 \% \text { of the } \\
\text { assemblies will be } \\
\text { between the minimum and } \\
\text { maximum gap. }\end{array}$ & $\begin{array}{l}99.73 \% \text { of the assemblies } \\
\text { will be between the } \\
\text { minimum and maximum } \\
\text { gap. The correction factor } \\
\left(C_{f}\right) \text { is a safety factor. }\end{array}$ \\
\hline
\end{tabular}




\subsubsection{Calculate the Variation for the Performance Requirement}

\subsubsection{Worst Case Model}

The following equation calculates the expected variation at the gap [7-9]:

$$
t_{w c}=\sum_{i=1}^{n}\left|a_{i} t_{i}\right|
$$

The minimum and maximum gaps are equal to:

$$
\begin{aligned}
& \text { Minimum Gap }=d_{g}-t_{w c} \\
& \text { Maximum Gap }=d_{g}+t_{w c}
\end{aligned}
$$

If either the value of minimum gap or maximum gap doesn't conform to the requirement, then the tolerance value of each component in the assembly needs to be resized by using:

$$
F_{w c}=\frac{d_{g}-g_{m}-\sum_{j=1}^{p}\left|a_{j} t_{j f}\right|}{\sum_{k=1}^{q}\left|a_{k} t_{k v}\right|}
$$

The resize factor is only applied to variable component by using:

$$
t_{k v, w c, \text { resized }}=F_{w c} \cdot t_{k v}
$$

Then expected variation after resizing is:

$$
t_{w c, \text { resized }}=\sum_{j=1}^{p}\left|a_{j} t_{j f}\right|+\sum_{k=1}^{q}\left|a_{k} t_{k v, w c, \text { resized }}\right|
$$

So the new minimum and maximum gaps are:

$$
\begin{aligned}
& \text { Minimum Gap }=d_{g}-t_{w c, \text { resized }} \\
& \text { Maximum Gap }=d_{g}+t_{w c, \text { resized }}
\end{aligned}
$$

\subsubsection{RSS Model}

The expected variation is calculated by [7-9]:

$$
t_{r s s}=\sqrt{a_{1}^{2} t_{1}^{2}+a_{2}^{2} t_{2}^{2}+a_{3}^{2} t_{3}^{2}+\ldots+a_{n}^{2} t_{n}^{2}}
$$

The minimum and maximum gaps are equal to: 


$$
\begin{aligned}
& \text { Minimum Gap }=d_{g}-t_{r s s} \\
& \text { Maximum Gap }=d_{g}+t_{r s s}
\end{aligned}
$$

If either the value of minimum gap or maximum gap doesn't conform to the requirement, then the tolerance value of each component in the assembly needs to be resized by using:

$$
F_{r s s}=\sqrt{\frac{\left(d_{g}-g_{m}\right)^{2}-\sum_{j=1}^{p}\left(a_{j} t_{i f}\right)^{2}}{\sum_{k=1}^{q}\left(a_{k} t_{k v}\right)^{2}}}
$$

The resize factor is only applied to variable component by using:

$$
t_{k v, r s s, r e s i z e d}=F_{r s s} \cdot t_{k v}
$$

Then expected variation after resizing is:

$$
t_{\text {rss, resized }}=\sum_{j=1}^{p}\left|a_{j} t_{j f}\right|+\sum_{k=1}^{q}\left|a_{k} t_{k v, r s s, \text { resized }}\right|
$$

So the new minimum and maximum gaps are:

$$
\begin{aligned}
& \text { Minimum Gap }=d_{g}-t_{r s s, \text { resized }} \\
& \text { Maximum Gap }=d_{g}+t_{r s s, \text { resized }}
\end{aligned}
$$

\subsubsection{MRSS Model}

The expected variation is calculated by [7-9]:

$$
t_{m r s s}=C_{f} \sqrt{a_{1}^{2} t_{1}^{2}+a_{2}^{2} t_{2}^{2}+a_{3}^{2} t_{3}^{2}+\ldots+a_{n}^{2} t_{n}^{2}}
$$

Where: $C_{f}=\frac{0.5\left(t_{w c}-t_{r s s}\right)}{t_{r s s}(\sqrt{n}-1)}+1$

The minimum and maximum gaps are equal to:

$$
\begin{aligned}
& \text { Minimum Gap }=d_{g}-t_{m r s s} \\
& \text { Maximum Gap }=d_{g}+t_{m r s s}
\end{aligned}
$$

If either the value of minimum gap or maximum gap doesn't conform to the requirement, then the tolerance value of each component in the assembly needs to be resized by using: 


$$
F_{m r s s}=\frac{-b-\sqrt{b^{2}-4 a c}}{2 a}
$$

Where:

$$
\begin{aligned}
a= & 0.25\left(\sum_{k=1}^{q} a_{k} t_{k v}\right)^{2}-2.25 \sum_{k=1}^{q}\left(a_{k} t_{k v}\right)^{2}+3 \sqrt{n} \sum_{k=1}^{q}\left(a_{k} t_{k v}\right)^{2}-n \sum_{k=1}^{q}\left(a_{k} t_{k v}\right)^{2} \\
b= & 0.5 \sum_{k=1}^{q}\left(a_{k} t_{k v}\right) \sum_{j=1}^{p}\left(a_{j} t_{j f}\right)+\left(\sum_{k=1}^{q} a_{k} t_{k v}\right)\left(d_{g}-g_{m}\right)-\sqrt{n}\left(\sum_{k=1}^{q} a_{k} t_{k v}\right)\left(d_{g}-g_{m}\right) \\
c= & 0.25\left(\sum_{j=1}^{p} a_{j} t_{j f}\right)^{2}+\left(d_{g}-g_{m}\right)^{2}-2 \sqrt{n}\left(d_{g}-g_{m}\right)^{2}+n\left(d_{g}-g_{m}\right)^{2} \\
& +\left(\sum_{j=1}^{p} a_{j} t_{j f}\right)\left(d_{g}-g_{m}\right)-\sqrt{n}\left(\sum_{j=1}^{p} a_{j} t_{j f}\right)\left(d_{g}-g_{m}\right)-2.25 \sum_{j=1}^{p}\left(a_{j} t_{j f}\right)^{2} \\
& +3 \sqrt{n} \sum_{j=1}^{p}\left(a_{j} t_{j f}\right)^{2}-n \sum_{j=1}^{p}\left(a_{j} t_{j f}\right)^{2}
\end{aligned}
$$

The resize factor is only applied to variable component by using:

$$
t_{k v, \text { mrss, resized }}=F_{m r s s} \cdot t_{k v}
$$

Then expected variation after resizing is:

$$
t_{\text {mrss }, \text { resized }}=\sum_{j=1}^{p}\left|a_{j} t_{j f}\right|+\sum_{k=1}^{q}\left|a_{k} t_{k v, \text { mrss }, \text { resized }}\right|
$$

So the new minimum and maximum gaps are:

$$
\begin{aligned}
& \text { Minimum Gap }=d_{g}-t_{\text {mrss, resized }} \\
& \text { Maximum Gap }=d_{g}+t_{\text {mrss, resized }}
\end{aligned}
$$

\subsection{Geometrical Tolerance Analysis}

Beside dimensional tolerance, geometrical tolerance in a component is also need to be analyzed in stacked tolerance. Geometric tolerance will control form, orientation and location of the feature. GDT controls are generally used only in worst case analysis. Since WC model assumes $100 \%$ inspection, so GDT control will influence the gap variation. In a statistical analysis, GDT doesn't influence to gap variation because manufacturing processes themselves are sources for variation. 
Rules in geometrical tolerance analysis [7,10,11]:

- Location control on a feature in the loop diagram is usually included in the analysis.

- Orientation control on a feature in the loop diagram is included in the analysis as long as the location of the feature is not a contributor to the requirement.

- Form control on a feature in the loop diagram is included in the analysis as long as the location, orientation or size of the feature is not a contributor to the requirement.

- Geometric form and orientation controls on datum features are usually not included in the loop diagram since datum is starting point for measurement and considered as TGC.

If form or orientation control is used in the loop diagram, then it is modeled with a nominal dimension equals to zero and an equal bilateral tolerance equals to form or orientation tolerance.

For location control at RFS, feature's size and location are treated independently. Meanwhile for MMC or LMC condition, the size and location dimension can't be treated independently.

For MMC or LMC condition, first it is necessary to calculate the largest outer boundary and smallest inner boundary allowed by the dimensions and tolerances. Formulas to calculate these boundaries can be seen at Table 2 .

Table 2 Formulas to calculate outer and inner boundary for location control at MMC or LMC condition [7].

\begin{tabular}{cccc}
\hline Feature & Condition & Inner Boundary & Outer Boundary \\
\hline External & MMC & LMC - Tolerance at LMC & $\begin{array}{c}\text { MMC + Geometric Tolerance } \\
\text { at MMC }\end{array}$ \\
& LMC & $\begin{array}{c}\text { LMC - Geometric Tolerance } \\
\text { at LMC }\end{array}$ & MMC + Tolerance at MMC \\
Internal & MMC & $\begin{array}{c}\text { MMC - Geometric Tolerance } \\
\text { at MMC }\end{array}$ & $\begin{array}{c}\text { LMC + Tolerance at LMC } \\
\text { LMC + Geometric Tolerance } \\
\text { at LMC }\end{array}$ \\
\hline
\end{tabular}

Next, convert the inner and outer boundary into a nominal diameter with an equal bilateral tolerance by using:

Nominal Diameter $=($ outer boundary + inner boundary $) / 2$

Equal Bilateral Tolerance $=($ outer boundary - inner boundary $) / 2$ 
Beside those three controls above, there is run-out control. Analyzing run-out control in tolerance stacks is similar to analyzing location control at RFS, where size and run-out tolerance are treated independently. Run-out tolerance can be modeled with a nominal dimension equals to zero and an equal bilateral equals to run-out tolerance/2.

And also there is concentricity control which is treated similar to location and run-out control. Concentricity tolerance can be modeled with a nominal dimension equals to zero and an equal bilateral equals to concentricity tolerance/2.

\section{Case Study}

Figure 2 shows a cross section of a Francis turbine assembly made by PT. GREAT. From this example, it will be demonstrated the stacked tolerance analysis.

On this example, there are several performance requirements, which are:

- Requirement 1. The gap between runner's cone and generator side cover must always be greater than zero to ensure that the runner can rotate freely but it shouldn't be too large to prevent leakage so the performance is still okay.

- Requirement 2. The gap between runner's ring and intermediate ring must always be greater than zero to ensure that the runner can rotate freely but it shouldn't be too large to prevent leakage so the performance is still okay.

- Requirement 3. The gap between guide vanes and generator side cover must always be greater than zero to ensure that the guide vanes can rotate freely but it shouldn't be too large to prevent leakage so the performance is still okay.

- Requirement 4. The gap between guide vanes and draft tube side cover must always be greater than zero to ensure that the guide vanes can rotate freely but it shouldn't be too large to prevent leakage so the performance is still okay.

Next, convert each requirement into an assembly gap requirement as follow:

- Requirement 1: $0<$ gap $1 \leq 0.6$.

- Requirement 2: $0<$ gap $2 \leq 0.6$.

- Requirement 3:0 $<$ gap $3 \leq 0.3$.

- Requirement 4: $0<$ gap $4 \leq 0.3$.

For this occasion, author only presents requirement 1 to represent requirement for radial rotor direction. Steps for analysis are described in following sub chapter. 


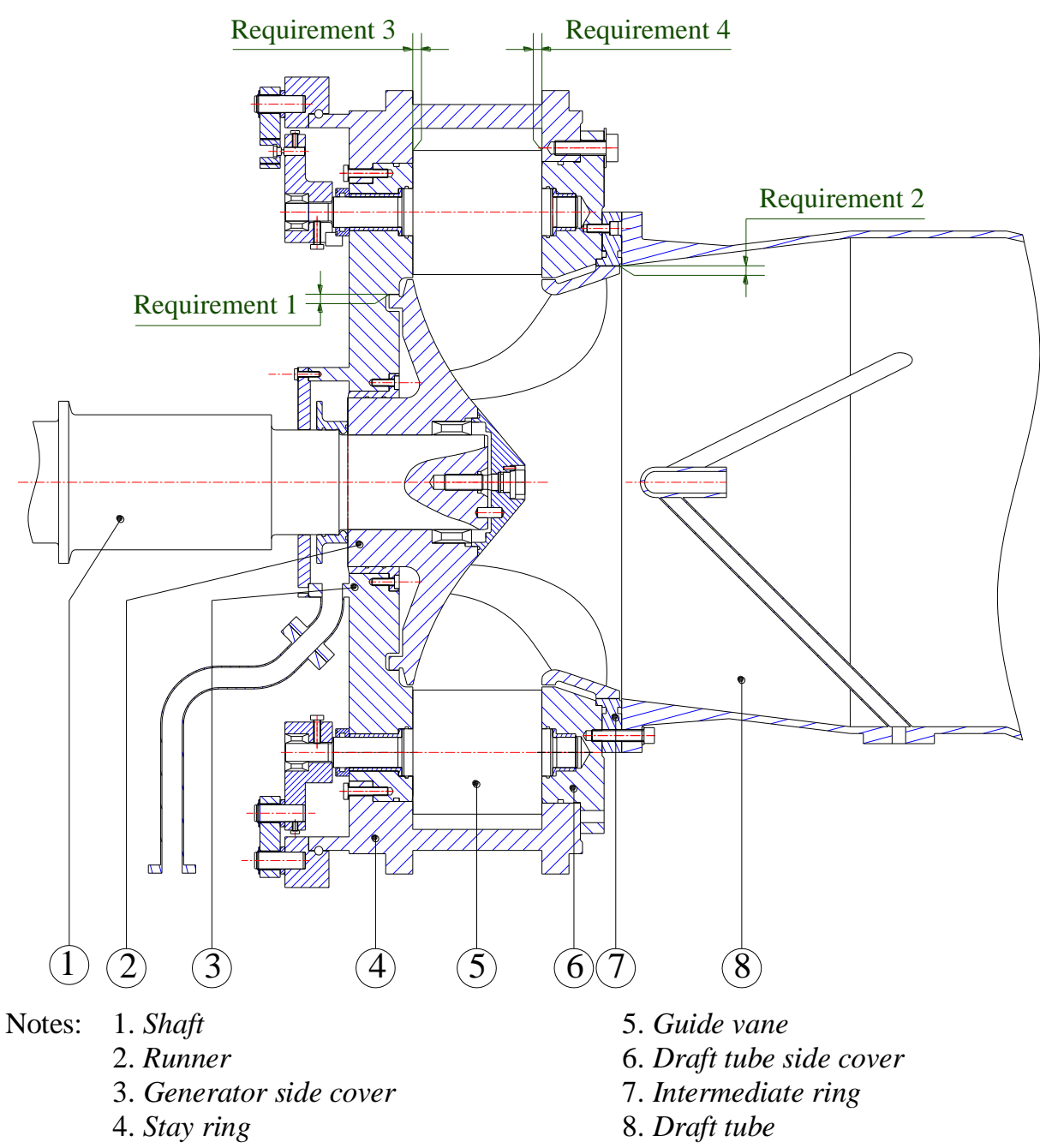

Figure 2 Cross Section of a Francis Turbine Assembly made by PT. GREAT.

\subsection{Establish the Performance Requirements}

Refers to performance requirement, gap 1 which is gap between runner's cone and generator side cover should be between $0 \mathrm{~mm}$ and $0.6 \mathrm{~mm}$.

\subsection{Drawing of a Loop Diagram}

It is necessary to determine base (or stopping point) so that the loop doesn't have to involve all components. For this analysis, assume turbine's stay ring (component number 4 in Figure 2) as the base. In analysis, base component is always considered ideal. So, in real, this stay ring must be manufactured and 
assembled properly to resemble ideal condition. Detail A on Figure 3 shows the loop diagram for gap 1.

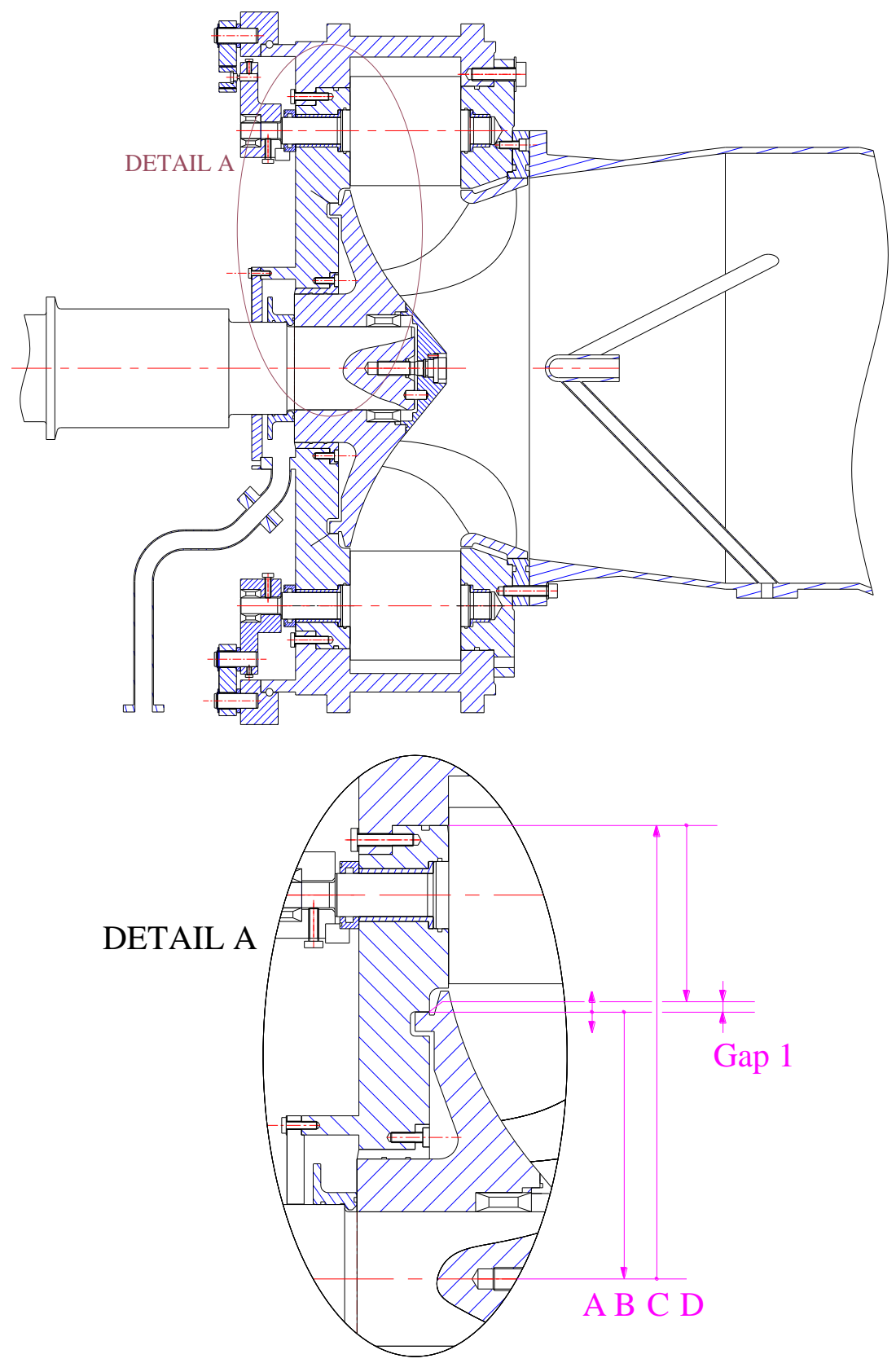

Figure 3 Turbine Assembly - Detail A. 
The explanations for each vector are as follow:

- $\mathrm{A}$ is a vector that represents concentricity tolerance of runner's cone. Its nominal value is 0 and its equal bilateral tolerance is half from concentricity tolerance. $\mathrm{A}=0 \pm 0.025 \mathrm{~mm}$. Its sensitivity factor is 1 . A is a variable component because its value can be adjusted by order.

- B is a vector that represents outside diameter of runner's cone. Its value is $556 \pm 0.05 \mathrm{~mm}$. Its sensitivity factor is -0.5 . B is a variable component because its value can be adjusted by order.

- $\mathrm{C}$ is a vector that represents outside diameter of generator side cover. Its value is $945 \mathrm{~g} 7\left(945_{-0.115}^{-0.025}\right) \mathrm{mm}$. Its sensitivity factor is 0.5 . $\mathrm{C}$ is a fixed component because it fits with another component so that it can't be changed to keep the performance.

- $\mathrm{D}$ is a vector that represents thickness of generator side cover until the gap. Its value is $945 \mathrm{~g} 7 \mathrm{~mm}-(557 \pm 0.05) \mathrm{mm}$. Its sensitivity factor is -0.5 . $\mathrm{D}$ is a variable component because its value can be adjusted by order.

\subsection{Converting All Dimensions to Mean Dimension with an Equal Bilateral Tolerance}

Table 3 shows the summary of vectors for gap 1 .

Table 3 Summary of Vectors for Gap 1.

\begin{tabular}{lccccc}
\hline \multicolumn{1}{c}{ Description } & Name & $\begin{array}{c}\text { Mean } \\
\text { Dimension }\end{array}$ & Sensitivity & $\begin{array}{c}\text { Fixed/ } \\
\text { Variable }\end{array}$ & $\begin{array}{c}\text { +/- Equal } \\
\text { Bilateral } \\
\text { Tolerance }\end{array}$ \\
\hline $\begin{array}{l}\text { Concentricity tolerance } \\
\text { of runner's cone }\end{array}$ & A & $0 \mathrm{~mm}$ & 1 & Variable & $0.025 \mathrm{~mm}$ \\
$\begin{array}{l}\text { Outside diameter of } \\
\text { runner's cone }\end{array}$ & B & $556 \mathrm{~mm}$ & -0.5 & Variable & $0.05 \mathrm{~mm}$ \\
$\begin{array}{l}\text { Outside diameter of } \\
\text { generator side cover }\end{array}$ & C & $944.93 \mathrm{~mm}$ & 0.5 & Fixed & $0.045 \mathrm{~mm}$ \\
$\begin{array}{l}\text { Thickness of generator } \\
\text { side cover }\end{array}$ & D & $387.93 \mathrm{~mm}$ & -0.5 & Variable & $0.095 \mathrm{~mm}$ \\
\hline
\end{tabular}

\subsection{Calculate the Mean Value for the Performance Requirement}

The mean value of the gap is: 


$$
\begin{aligned}
d_{g} & =\sum_{i=1}^{n} a_{i} \cdot d_{i} \\
d_{g} & =(1) A+(-0.5) B+0.5 C+(-0.5) D \\
d_{g} & =(1)(0)+(-0.5)(556)+(0.5)(944.93)+(-0.5)(387.93) \\
d_{g} & =0.5 \mathrm{~mm}
\end{aligned}
$$

\subsection{Determine the Method of Analysis}

For this case, worst case analysis model is chosen because PT. GREAT manufactures 1 turbine only and it is necessary to assure the correctness of each dimension $100 \%$.

\subsection{Calculate the Variation for the Performance Requirement}

The gap variation is:

$$
\begin{aligned}
t_{w c} & =\sum_{i=1}^{n}\left|a_{i} \cdot t_{i}\right| \\
t_{w c} & =|(1)(0.025)|+|(-0.5)(0.05)|+|(0.5)(0.045)|+|(-0.5)(0.095)| \\
t_{w c} & =0.12 \mathrm{~mm}
\end{aligned}
$$

So the maximum and minimum gaps are:

$$
\begin{aligned}
\text { Maximum gap } & =d_{g}+t_{w c} \\
& =0.5+0.12=0.62 \mathrm{~mm} \\
\text { Minimum gap } & =d_{g}-t_{w c} \\
& =0.5-0.12=0.38 \mathrm{~mm}
\end{aligned}
$$

As explained before in performance requirement, maximum gap must not exceed $0.6 \mathrm{~mm}$. So it is necessary to do an adjustment. To reach $0.6 \mathrm{~mm}$ maximum gap, the value of gap variation should be $0.1 \mathrm{~mm}$. So, the value of $g_{m}$ in Equation 4 must be $0.4 \mathrm{~mm}$. Then the resize factor is:

$$
\begin{aligned}
F_{w c} & =\frac{d_{g}-g_{m}-\sum_{j=1}^{p}\left|a_{j} t_{j f}\right|}{\sum_{k=1}^{q}\left|a_{k} t_{k v}\right|} \\
F_{w c} & =\frac{0.5-0.4-|(0.5)(0.045)|}{|(1)(0.025)|+|(-0.5)(0.05)|+|(-0.5)(0.095)|} \\
F_{w c} & =0.79487
\end{aligned}
$$


This resize factor is multiplied to each variable's tolerance. So the new values are:

$$
\begin{aligned}
& A=0 \pm 0.02 \mathrm{~mm} \\
& B=556 \pm 0.04 \mathrm{~mm} \\
& C=944.93 \pm 0.045 \mathrm{~mm} \\
& D=387.93 \pm 0.075 \mathrm{~mm}
\end{aligned}
$$

The gap variation after adjustment is:

$$
\begin{aligned}
t_{w c, \text { resized }} & =\sum_{i=1}^{n}\left|a_{i} \cdot t_{i}\right| \\
t_{w c, \text { resized }} & =|(1)(0.02)|+|(-0.5)(0.04)|+|(0.5)(0.045)|+|(-0.5)(0.075)| \\
t_{w c, \text { resized }} & =0.1 \mathrm{~mm}
\end{aligned}
$$

Then the maximum and minimum gaps are:

$$
\begin{aligned}
\text { Maximum gap } & =d_{g}+t_{w c} \text { resixed } \\
& =0.5+0.1=0.6 \mathrm{~mm} \\
\text { Minimum gap } & =d_{g}-t_{w c, \text { resized }} \\
& =0.5-0.1=0.4 \mathrm{~mm}
\end{aligned}
$$

\section{$4 \quad$ Result}

According to this analysis, design from PT. GREAT can't meet the performance requirement. Design from PT. GREAT creates gap 1 that varies between $0.38-$ $0.62 \mathrm{~mm}$. This fault can cause a decrease of turbine's efficiency and also difficulty in assembly process.

To fulfill the performance requirement 1 which demands $0<$ gap $1 \leq 0.6 \mathrm{~mm}$, components that build the assembly must have dimensions and tolerances as shown in Figure 4.

From this analysis, it can be concluded that gaps in assembly can be controlled since design process. So designers must consider this gap analysis to assure product's performance and assemblability. 

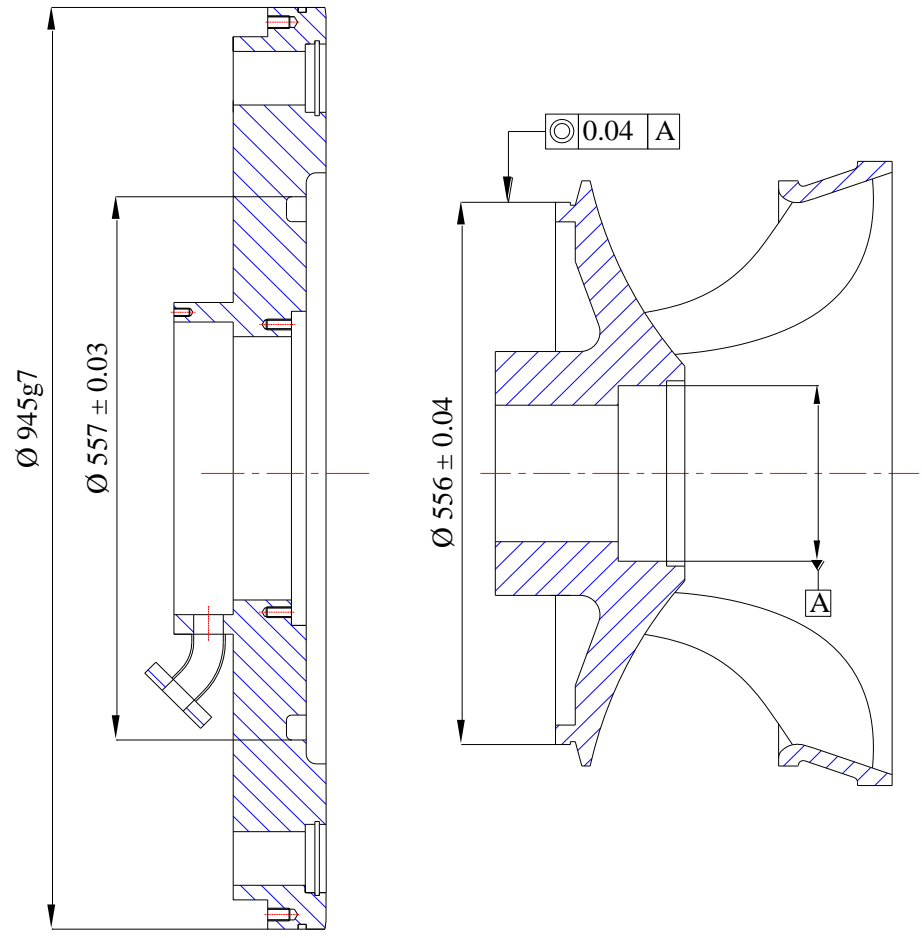

Figure 4 Dimensions and Tolerances of Radial Components (Gap 1) After Adjustment.

\section{Nomenclature}

$a_{i}=$ sensitivity factor that defines direction and magnitude for the $i^{\text {th }}$ dimension

$a_{j}=$ sensitivity factor for the $j^{\text {th }}$, fixed component in the stack up

$a_{k}=$ sensitivity factor for the $k^{\text {th }}$, variable component in the stack up

$C_{f}=$ correction factor used in MRSS equation

$d_{i}=$ the mean value of the $\underline{i}^{\text {th }}$ dimension in the loop diagram

$d_{g} \quad=$ the mean value at the gap (positive means clearance and negative means interference)

$F_{m r s s}=$ resize factor for MRSS model

$F_{r s s}=$ resize factor for RSS model

$F_{w c}=$ resize factor for $\mathrm{WC}$ model 


$$
\begin{aligned}
g_{m} & =\text { minimum value at the gap } \\
& =0 \text { if no interference or clearance is allowed } \\
n & =\text { the number of independent variables (dimensions) in the stack up } \\
p & =\text { number of independent, fixed dimension in the stack up } \\
q & =\text { number of independent, variable dimension in the stack up } \\
t_{i} & =\text { equal bilateral tolerance of the } i^{\text {th }} \text { component in the stack up } \\
t_{j f} & =\text { equal bilateral tolerance of the } j^{\text {th }} \text {, fixed component in the stack up } \\
t_{k v} & =\text { equal bilateral tolerance of the } k^{\text {th }}, \text { variable component in the } \\
t_{m r s s} & =\text { maximum expected variation (equal bilateral) using MRSS model } \\
t_{r s s} & =\text { maximum expected variation (equal bilateral) using RSS model } \\
t_{w c} & =\text { maximum expected variation (equal bilateral) using WC model }
\end{aligned}
$$

\section{References}

[1] Investor Daily Friday, JAKARTA, 23 February 2007.

[2] Bernhard Pelikan (President ESHA), European Small Hydro Power Association, Guide on How to Develop a Small Hydropower Plant - Part 1, ESHA Publishing, 2004.

[3] Bernhard Pelikan (President ESHA), European Small Hydro Power Association, Guide on How to Develop a Small Hydropower Plant - Part 2, ESHA Publishing, 2004.

[4] Hartwig Petermann, Stroemungsmaschinen, Springer Verlag, Berlin Heidelberg - New York, 1972.

[5] Bernhard Pelikan, The President of European Small Hydropower Association, Guide on How to Develop a Small Hydropower Plant, ESHA, 2004.

[6] Arne Kjølle, The Development of Hydropower in Norway, Trondheim, 2001.

[7] Paul Drake, JR., Dimensioning and Tolerancing Handbook, McGrawHill, ISBN 0-07-018131-4, 1999.

[8] Georg Henzold, Geometrical Dimensioning and Tolerancing for Design, Manufacturing and Inspection, Elsevier Publishing, ISBN-13: 978-07506-6378-8, 2006.

[9] Hong-Chao Zhang, Advanced Tolerancing Techniques, Willey Interscience, ISBN 0-471-14594-7, 1997. 
[10] Gene R. Cogorno, Geometric Dimensioning and Tolerancing for Mechanical Design, McGraw-Hill, 2006.

[11] Bryan R. Fischer, Mechanical Tolerance Stackup and Analysis, Marcel Dekker Inc. 